\title{
New Insights into HIV/AIDS for Students and Healthcare Professionals
}

\author{
OMOBUWA Olubukunola
}

"New Insights into HIV/AIDS for Students and Healthcare Professionals" (Eds. Esther Olufunmilayo Asekun-Olarinmoye and Christopher Olutayo Alebiosu) is a textbook written by a total of 40 authors who are specialists in diverse fields of biomedical research and medical practice; and are based in well-established higher institutions of learning in Nigeria. The book was published by Cambridge Scholars Publishing, United Kingdom in 2019. The 27-chaptered textbook (with topics ranging from "History of HIV" to "Policy and Advocacy" in HIV/AIDS) has articulately presented the most important and relevant information on all aspects of HIV and AIDS in a well-organized fashion and in simple, scientific language which can be easily read and understood by a wide variety of audience ranging from medical students to diverse cadres/categories of health personnel, including specialists. The lay public will also find this book, which is dedicated to stakeholders in HIV care, very useful.

The preface of the textbook was written by the current Vice-Chancellor of the Osun State University, Professor Labode Popoola; and the foreword by Professor C. O. Alebiosu who at the time of publication was on sabbatical as Visiting Professor of Medicine at Yale University Office of Global Health, from where he served as Visiting Medical Professor/Consultant at the Liberian College of Physicians and Surgery.

Human Immunodeficiency Virus (HIV) chronic and potentially life-threatening condition called acquired immunodeficiency syndrome, or AIDS. HIV/AIDS and is a major public health concern and cause of death in many African countries (1).

The first chapter of the textbook gives a concise history of HIV and did not fail to mention its origin, as being from the non-human primates in Central and West Africa, types (HIV-1 and HIV-2) and year of the first recognition of the Acquired Immunodeficiency Syndrome (AIDS), a condition caused by HIV, i.e. $1981(2,3)$. HIV/AIDS has continued to be a menace globally and especially in sub-Saharan Africa where it was ranked the $5^{\text {th }}$ cause of death in the southern part of the region in 1990 and rose to become the top cause of death in the same region only 20 years later (2010).

The second chapter took a dive into the epidemiology of HIV where it was unequivocally stated that HIV has attained a pandemic proportion and that the following categories of individuals significantly fuel its spread: "men having sex with men (MSM), intravenous drug users and those involved in commercial sex transactions (works). In sub-Saharan Africa however, heterosexual transmission as well as blood transfusion involving HIV-infected donors also play important roles in HIV transmission". The modes of HIV transmission as well as important determinants/vulnerability factors, including risk level associated with each mode of transmission were described in detail. In the same chapter, relevant figures for the years 2010 and 2015 such as the number of people living with HIV

Department of Community Medicine, Osun State University, Osogbo.

http://orcid.org/0000-0002-6315-4686

E-mail: olubukola.omobuwa@uniosun.edu.ng 
(PLHIV) [all ages], new HIV infections [all ages], PLHIV on antiretroviral treatment [all ages] and AIDS-related deaths [all ages] were also compared. The following were also documented: ..... that in virtually all regions of the globe, there is an increase in the number of PLHIV (apparently due to increased survival as more people are on ARVs) and a decrease in "New HIV Infections" between 2010 and 2015;... there is an upsurge in the number of "PLHIV on antiretroviral treatment" and a decrease in deaths associated with AIDS infections. Concerning the percentage coverage of antiretroviral therapy globally, just over one-half of people with HIV are receiving the needed drugs (4). However, the section of this chapter which addressed "distribution" of HIV/AIDS is rather brief and could be improved by providing more information on distribution with respect to the trend of the disease over time in different global regions, and especially in Africa. It is also crucial to describe the impacts of the disease across the globe over time, for example, there was a surge in the prevalence of orphans and vulnerable children in Southern Africa as a result of HIV infections.

The chapter on "Virology of HIV" provides a detailed review of the literature on the structure, genetic diversity and lifecycle of HIV details of which are essential, as they serve to allow for the identification of important aspects of the lifecycle of HIV thus providing insight into the development of novel methods for diagnosis of HIV as well as highlighting potential targets for antiviral therapy and vaccination against HIV. The chapter was concluded with following profound statements: "HIV-1 is incontrovertibly one of the most studied infectious agents of the last three decades. The availability of new molecular diagnostic technologies has allowed further insight into the HIV structure and replication. Furthermore, reports from various studies have highlighted the different viral subtypes and recombinant forms as well as the significance of such differences in the infection cycle. The current chapter on the virology of HIV provides important information which is useful for the design of novel and therapeutic approaches aimed at interrupting the HIV life cycle".

Majority of the subsequent chapters focused on the typical presentations/manifestations of HIV in relation to the various systems of the human body. Haematological presentations of HIV discussed in detail include anaemia (there are many causative factors for this in HIV infection and they are described in detail), thrombocytopaenia (grossly decreased platelet count below normal levels), leukopaenia, bone marrow diseases, and coagulation disorders. However, this chapter has many short paragraphs, several of which can be merged together. A chapter which addressed neurocognitive disorders associated with HIV elaboratively discussed asymptomatic neurocognitive impairment (ANI), mild neurocognitive disorder (MND) and HIVassociated dementia (HAD); the clinical features of each of the medical alterations were well-presented.

Complications of HIV infection were presented in a chapter which established that such complications are multi-systemic in nature and they may be the mode of first-time presentation of a previously undiagnosed HIV positive patient (5). Complications of HIV are known to be due to the chronic nature of the disease and they may sometimes be the result of therapeutic effects. This chapter could be improved upon by providing some more details on each of the said complications listed and including some other complications not included (e.g. respiratory system, opportunistic infections). The chapter on 'HIV and the eye' pointed out that the incidence of blindness as a result of HIV infection has declined; diverse eye manifestations (and their suggested management) both in adults and children were discussed in detail. I suggest that the subheading "How does HIV affect the eye?" be changed from question format to a statement format.

Otolaryngologic manifestations of HIV infection were succinctly classified and explicitly discussed in the eighth chapter of the book where it was clearly stated that "HIV testing should be offered to patients with lymphadenopathy of unknown cause" as an enlarging mass is the major manifestation of HIV in the neck region. The authors of this chapter stressed the need for all clinicians to familiarize themselves with the otolaryngologic manifestations of HIV in order to help facilitation of early recognition and prompt institution of management. The author of the chapter on HIV and the respiratory system opened the chapter with a statement of fact that "the respiratory system is the most frequently affected organ-system by HIV infection and respiratory pathology is most commonly associated with morbidity and mortality among patients living with HIV/AIDS" (6) and also mentioned that adherence to a regimen of an effective combination of HAART leads to a containment of HIV multiplication, and preservation or improvement in immunological function, with a 
reduced incidence of opportunistic infections, morbidity and mortality; recommended investigations in the event of a respiratory manifestation of HIV were discussed and radiographs that may be helpful for diagnosis were included in the chapter.

In the chapter on HIV/AIDS and the cardiovascular system, the authors in their opening statement wrote without mincing words that "both HIV infection, on a long-term basis, and the side effects of its treatment with Highly Active AntiRetroviral Therapy (HAART) cause cardiovascular diseases (CVD)"; a statement one could describe as scary as one may tend to think that CVD is inevitable in HIV/AIDS patients and they went on to mention that "the pathogenesis of CVD in HIV infection is likely to be multifactorial". Diverse cardiovascular manifestations on HIV/AIDS, investigations and treatments were outlined and they took a dive into future perspectives stating that "as HIV-infected patients live longer, HIV-related cardiovascular disease will become the commonest cause of mortality and a vital area of research"; the authors however also stated that "commencement of early treatment with ART is targeted as a likely strategy to reduce CVD risk for patients infected with HIV" (7) and also that a good understanding of genetic predispositions to QT prolongation may serve as a useful guide to treatment and that understanding the aetiologies of cardiomyopathy may be beneficial to various kinds of research efforts, such as the effects of cytokine, mitochondrial, and neurohormonal pathways.

The chapter on HIV and the gastrointestinal system (GIT) describe an array of GIT manifestations of HIV ranging from HIV enteropathy to esophageal motility disorder. Complications, such as liver dysfunction, GIT malignancies (Kaposi's sarcoma and Non-Hodgkin's lymphoma) were also discussed. The authors concluded with comforting statements that "the introduction of HAART has drastically reduced opportunistic aetiologies, along with HIV enteropathy" and that "HAART not only improves the systemic immune system but also the local cellular immunity of the GIT".

The authors of the chapter on renal diseases in HIV/AIDS stated clearly that "renal involvement can occur at all stages of HIV infection and can be the initial clue to the presence of HIV infection in an undiagnosed patient but HIV-associated nephropathy (HIVAN) is one of the syndromes directly caused by HIV infection" (8). HIVAN was said to be one of the chronic kidney diseases directly caused by HIV infection, that it is commoner among blacks and is the third leading cause of end-stage renal disease. A chapter which reviewed the prevalence, risk factors, clinical features, diagnosis and management of DM in HIV-infected individuals recognized that the occurrence of diabetes mellitus (DM) in Human Immunodeficiency Virus (HIV)-infected patients doubles the challenge of managing such individuals. The authors concluded that diabetes coexisting with HIV infection is quite challenging but manageable and the outcome is good if promptly diagnosed and properly managed. The authors of the chapter titled "HIV and reproductive health" identified poverty, women's lack of power to take decisions by themselves and for themselves over their sexual and reproductive lives, and gender-based violence and discrimination as the challenges worsening the HIV/AIDS epidemic; and went ahead to discuss the inter-relationships between HIV/AIDS and reproductive health services. The chapter gave a background synopsis of what reproductive health is all about and then went on to elaborate on how it relates/links with HIV/AIDS. Several aspects of the relationships between both subjects (such as HIV and family planning services, pregnancy, breastfeeding, circumcision, etc.) were explored in detail.

Another chapter which discussed HIV/AIDS and musculoskeletal disorders said about $72 \%$ of HIV/AIDS patients suffer musculoskeletal disorders of various types ranging from arthralgia to osteomyelitis as well as neoplasms. Infectious and non-infectious musculoskeletal disorders were explicitly discussed and the authors concluded by iterating that HIV/AIDS progressively eliminates CD4+ T-helper lymphocytes and permits opportunistic infections and tumours that compromise the three principal components: the joint (cartilages, tendons and ligaments), the muscle (soft tissue) and the bone (osseous tissue) of the musculoskeletal system leading to a variety of disorders involving the musculoskeletal system. Therefore, adequate knowledge of the existence and the characteristic pathological appearance of the conditions affecting osseous tissue, articular cartilages, and muscle (soft tissues) in HIV/AIDS individuals using radiological imaging techniques specific for each pathological condition is valuable for diagnosis, detection, and the appropriate treatment regimen in HIV infections.

The author of "skin manifestations of HIV infection" unequivocally stated that "skin disorders 
are quite common and usually more aggressive in human immunodeficiency virus (HIV)-positive patients than the general population" (9) and went on to describe the diverse types and spectrum of skin disorders associated with HIV infection. The author said "Clinicians should be familiar with the wide variety of mucocutaneous manifestations in HIVinfected persons as they commonly have cutaneous abnormalities; with the prevalence of such mucocutaneous manifestations approaching 100\%". The author concluded with the following statements: "HIV infection should be suspected when a cutaneous lesion tends to be chronic, severe, bizarre, or involving more than one dermatome. Physicians caring for People Living with HIV should be familiar with the diagnosis and management of common cutaneous lesions because prompt and appropriate management of these conditions will reduce morbidity".

A previous study found Pseudomembranous candidiasis to be the commonest oral manifestation of HIV/AIDS, according to the chapter considering that topic (10). Several other oral lesions associated with HIV/AIDS were discussed, including Kaposi's sarcoma and Non-Hodgkin's Lymphoma. Dry mouth (xerostomia) was also listed as a possible oral manifestation of HIV/AIDS. The relationship (inverse) between CD4 count and prevalence of oral lesions in HIV/AIDS patients was described and the authors also stated that high prevalence of oral lesion is associated with low CD4 counts. However, the conclusion of this chapter needs review as it is rather long and also contains portions that should have come up in the main body of the write-up (e.g. the opening statement is actually reporting the finding of a previous study). The next chapter titled "HIVassociated malignancies" made the following profound statements: "People Living With HIV/AIDS (PLWHA) are at increased risk of developing some malignancies. These AIDSassociated malignancies constitute major complications encountered by HIV/AIDS patients upon immune suppression. A weak immune system itself may aid carcinogenesis and spread some cancers more rapidly, more so when conventional cancer management methodologies such as chemotherapy and radiation therapy can also weaken the immune system". The chapter further classified and described HIV-associated cancers with respect to their clinical features, risk factors, diagnosis (including screening) and treatment. The author also suggests modalities for the prevention and control of HIV-associated malignancies.
The paediatric population was not left out in this book as a whole chapter was dedicated to the topic "HIV/AIDS and the paediatric population". The authors' opening statement categorically paints a clear picture of the unfavourable and precarious situation of the African children as far as HIV/AIDS is concerned: "The children of Nigeria and subSaharan Africa have borne a disproportionate burden of the scourge of the pandemic of paediatric HIV infection". The chapter highlighted the history, epidemiology, clinical presentation, psychosocial aspects, control efforts, recent advances and future directions. Mother-to-child transmission (MTCT) is by far the commonest mode of transmission to children and it accounts for more than of transmission in children and can occur in-utero, at delivery or during breastfeeding. One of the pertinent points made in this chapter is that various studies have shown that, compared to HIV unexposed children, HIV-exposed but uninfected children (HEU) have greater low birth weight rates, a higher prevalence of growth, development and immunologic abnormalities and ultimately higher mortality rates. The authors then mentioned that "theories proffered to explain these outcomes include the side effects of ARVs taken by the mother and the baby, prevalent food insecurity in many HIV-affected households and lately, coexisting maternal cytomegalovirus infection (maternal HIV-cytomegalovirus coinfection) which was transmitted to the baby". The chapter also puts on record that early infant diagnosis (EID) is available in Nigeria and stated as follows: "The Nigerian EID program aims to provide EID services for at least $90 \%$ of all HIV-exposed infants and targets to test more than 158,000 HIV-exposed infants by 2020 in line with the UNAIDS 90-90-90 global target" (11) However, it is sad to note that "so far, EID coverage has remained low with only $4 \%$ of HIV-exposed infants receiving a virologic test within two months of birth". The concept of elimination of mother-to-child transmission (EMTCT) which is the global commitment that provided the impetus to achieving a Millennium Development Goal target of mother-to-child transmission of HIV. The authors then concluded as follows: "The Millennium Development Goal of reversing the HIV epidemic was reached ahead of the 2015 deadline - an incredible achievement that testifies to the power of national action and international solidarity. The progress achieved in the past 15 years should galvanize the global community to build on the momentum and end paediatric HIV infections by $2030 "$. 
On the subject of HIV and pregnancy, the authors wrote: "The natural course of HIV/AIDS has not been known to be affected significantly by pregnancy. There is no evidence that pregnancy accelerates the progression from HIV to AIDS". A very important take-away from this chapter in relation to the recommended mode of delivery for HIV positive pregnant women. The authors stated that "routine elective cesarean section is no longer practiced. The vaginal route is used in women who are on ARV (HAART or single agent), whose viral load is less than 1000 copies $/ \mathrm{mm}^{33}$." However, for those whose viral load is $\geqslant 1000$ copies $/ \mathrm{mm}^{3}$ caesarean section may be used especially in advanced countries. Antenatal care, labour and post-partum care management are well discussed. There is a need to review the font size of the sub-headings contained in this chapter as well as correct a few errors in the use of upper- and lower-case letters within sentences. A section of the chapter reads "Caesarian Section in HIV women"; the word "positive" should be placed after HIV, and in the sub-heading "Management of a discordant couple, 'HIV-' should be inserted just before the word 'discordant'. The chapter that discussed the neurological manifestations of HIV/AIDS established that neurological disease is the first manifestation of HIV infection in about $20 \%$ of patients; in advanced disease, about $60 \%$ of patients will have a clinically evident neurologic disorder and up to $90 \%$ of patients with advanced disease have pathologic abnormalities of the nervous system at autopsy. CNS and PNS disorders that may occur in HIV infection were elaborately discussed. However, many short paragraphs which can be merged together are contained in this chapter.

The chapter on HIV/AIDS and mental health established that psychosocial issues have been intimately related to HIV infection since it was discovered in 1981 and therefore set out to explore the implications of recent advances made in the treatment of HIV/AIDS for the mental health of those affected. In addition, the diagnosis and treatment of mental disorders in people living with HIV and AIDS were discussed and the author also looked into the availability of mental health care and barriers to the care of the mental health of people living with HIV/AIDS, especially in the low- and middleincome countries. The author further stated that studies have established that treatment of mental health problems in patients with HIV improves the outcome. Good counselling is known to be very helpful in HIV infection as it is known to promote behavioural change and optimal mental health, and helps them to lead more positive lives as well as prevent further transmission of HIV. The intricacies of counselling processes are aptly described, relevant ethical principles as well as barriers to effective HIV counselling and testing are also highlighted in the chapter.

Pharmacological treatment of HIV/AIDS has been the mainstay of managing People Living with HIV (PLHIV) for nearly four decades of the existence of the disease which has hitherto defied any cure. "The goals of ARV are to reduce morbidity and prolong the life of PLHIV, to achieve a rapid and sustained suppression of the viral load, as well as to facilitate their ability to live positively and enjoy an improved quality of life". The authors mentioned the "HIV Test and Start" approach which was commenced in 2017 and also clearly highlighted the donor-dependency nature of the availability of ARVs in developing countries. The goals of ART and mechanisms of action of ARVs are clearly spelt out in this chapter. The diverse classes and combinations of ARVs are elaborated and paediatric ART is aptly described. A section of the chapter briefly discusses the future of ARVs and the feasibility of HIV vaccines. Reference was made to the "Berlin patient" who seems to be the only person ever cured of HIV till date. In the chapter which discussed laboratory investigations a review to identify laboratory investigation tools and approaches that are currently available for the ease of diagnosis and management of HIV/AIDS patients is succinctly presented. Questions on who to be tested, what tests should be conducted, timing of testing, and what other steps to take in conjunction with testing (pre- and post-test counselling) are answered. A key aspect of this chapter is the inclusion of investigations to identify resistance to ART. I however suggest that subheadings presented in question format be converted into statement format.

"HIV/AIDS, being a cross-cutting issue that has multi-sectorial involvement having various impacts in a country, needs policies that would guide the robust response to its menace in a given population; .... being a cross-cutting issue makes it impossible for any government alone in a country to control its spread and effect; there is a need for other actors to advocate various stakeholders and the general population on how best way it can be controlled" (excerpts from the chapter on "policy and advocacy in HIV/AIDS). The authors of this final chapter of the book also opined that "the policy field of HIV/AIDS 
globally has many players with competing interests and different levels of influence". HIV Prevention 2020 Roadmap was presented and discussed. Policy instruments for Nigeria on HIV were highlighted and discussed; the role of advocacy in formulation of policies at national and global levels were also explored. The authors concluded by implying that policy and advocacy in HIV/AIDS response is an essential process for the global accomplishment of the goal $90: 90: 90$ by 2020, and especially for Nigeria which has a high burden of the disease.

I observed certain inconsistencies in the textbook such as formatting of the chapter headings (some have varying font sizes for words contained therein while others have uniform font sizes); also, some chapters finished with a conclusion while others do not. It would be great to follow a uniform format for all the chapters, including positioning and pattern of stating sub-headings (many of the subheadings are incomplete e.g. "history" on page 103, which should actually have been written as "history-taking in a patient with suspected respiratory manifestation of HIV". Another observation in this textbook is that in some chapters, pictures (figures) are presented within the write-up whereas they were presented at the end in most of the other chapters. Uniformity in mode of presentation of figures is desirable; also, the use of coloured photographs/figures is more preferable when compared with the choice of black and white utilized in the book. There are also a number of grammatical errors which can be corrected.

A very important concern about this textbook is its market price, $\$ 119.95$ on Amazon which translates to 43,182 naira (Nigerian currency). This is a huge obstacle to its affordability to the target audience, especially those living in resource poor settings, and particularly in sub-Saharan Africa. The editors will do well to look into the possibility of producing a more affordable version when considering the production of a revised edition (which is expected to address issues raised and observations made in this review and of course, provide relevant updates).

By and large, the authors of this "rich" textbook on a visibly significant, long-standing health menace of a pandemic proportion which will no doubt find great usefulness to students, teachers/lecturers, scientists, governments and even lay people, have done a commendable job for championing and charting pathways for the diffusion of the much sought-after knowledge needed for the containment and control of HIV/AIDS, a scourge which has plagued humanity for over four decades, and have smartly done the needful in this concise, single piece of textbook.

\section{REFERENCES}

1. Joint United Nations Programme on HIV/AIDS (UNAIDS); The Gap Report. ISBN: 978-92-9253062-4; 2014. Available at https://www.unaids.org/ sites/default/files/media_asset/UNAIDS_Gap_repo rt_en.pdf(Accessed on 12 June 2020).

2. CDC. Kaposi's sarcoma and Pneumocystis pneumonia among homosexual men - New York City and California. MMWR Morb Mortal Wkly Rep. 1981; 30: 305-308.

3. Greene WC. A history of AIDS: Looking back to see ahead. Eur J Immunol. 2007; 37 (Suppl. 1): S94-S102.

4. WHO. HIV AIDS Data and Statistics. Available at https://www.who.int/hiv/data/en/ (Accessed on 12 June, 2020).

5. Centre for Disease Control and Prevention (CDC). HIV Prevalence estimates - United States, 2006. MMWR Morb Mortal Wkly Rep. 2008; 57(39): 1073-1076.

6. Lanjewar DN, Duggal R. Pulmonary pathology in patients with AIDS: an autopsy study from Mumbai. HIV Med. 2001; 2: 266-271.

7. Longenecker CT, Triant VA. Initiation of antiretroviral therapy at high CD4 cell counts: does it reduce the risk of cardiovascular disease? CurrOpin HIV AIDS.2014;9(1):54-62.

8. Herman ES, Klotman PE. HIV-associated nephropathy: epidemiology, pathogenesis, and treatment. SeminNephrol. 2003; 23: 200-8.

9. Goldstein B, Berman B, Sukenik E, Frankel SJ. Correlation of skin disorders with CD4 lymphocyte counts in patients with HIV/AIDS. J Am Acad Dermatol. 1997; 36: 262-264.

10. Berberi A, Noujeim Z, Aoun G. 2015. Epidemiology of Oropharyngeal Candidiasis in Human Immunodeficiency Virus/Acquired Immune Deficiency Syndrome patients and CD4+ Counts. J Int Oral Health, 7(3): 20-23.

11. National Agency for the Control of AIDS, Federal Republic of Nigeria. Global AIDS Response Country Progress Report Nigeria: GARPR 2015. Available at: <http://www.unaids.org/sites/default/ files/en/dataanalysis/knowyourresponse/countrypro gressreports/2014countries/NGA_narrative_report_ 2015.pdf. Accessed on 26th May, 2020. 